\title{
Prédétermination de la durée de vie de barrages en Algérie
}

\author{
Predermination of the reservoirs life in Algeria
}

par M. Kassoul

Laboratoire de Mécanique

Université de Caen

Yearly the deposition of sediments amputates from $2 \%$ to $5 \%$ of the global water reserve of the hydraulic infrastructure in the Maghreb countries. The aim of the present study is to contribute to the deposit quantification in order to define proper hydrotechnical structures, and to increase their life time (reducing coasts).

Firstly, the author undertakes the information stemming from topo-bathymetric processing of 19 dams in Algeria. He therefore analyses these data with the aim of setting up a simple model for estimating deposits and classifying the Algerian dams.

\section{INTRODUCTION}

Le calcul de la sédimentation qui va s'accumuler dans une retenue est considéré comme étant un outil de prévision lors de la planification, la conception et l'exploitation d'un ouvrage hydraulique. La genèse, les transports et les dépôts des sédiments sont des processus dynamiques complexes : ils englobent l'érosion des sols, le dépôt aux pieds des versants, la substitution des charges, le sapement et l'effondrement des berges, le transport dans le réseau hydrographique par le charriage de fond, la transformation et l'eutrophisation des cuvettes, l'ensablement des estuaires et la formation des deltas. La figure 1 montre la genèse et le cheminement des sédiments dans les cours d'eau et bassins versants.

Ces processus restent difficiles à quantifier individuellement. Par contre, la mesure des sédiments au niveau des barrages est un résultat intégrant tous ces processus [1].

L'objectif est, après exploitation des résultats de quantification des alluvions des ouvrages envasés, de déterminer l'année limite de mise en service de nouveaux ouvrages hydrotechniques.

\section{CARACTÉRISTIQUES HYDROMORPHO- MÉTRIQUES DES SYSTÈMES ÉTUDIÉS}

Les différents barrages étudiés sont situés dans des bassins versants répartis sur une zone qui couvre une partie importante du territoire algérien, de par son relief varié (montagne, plaine), la diversité de sa végétation (forêt, broussailles, prairies,...) et de son climat (sub-humide, semi-aride, aride).

La majeure partie des bassins versants a une pluviométrie moyenne interannuelle comprise entre 300 et $600 \mathrm{~mm}$, le climat semi-aride y est fortement représenté. Cinq barrages

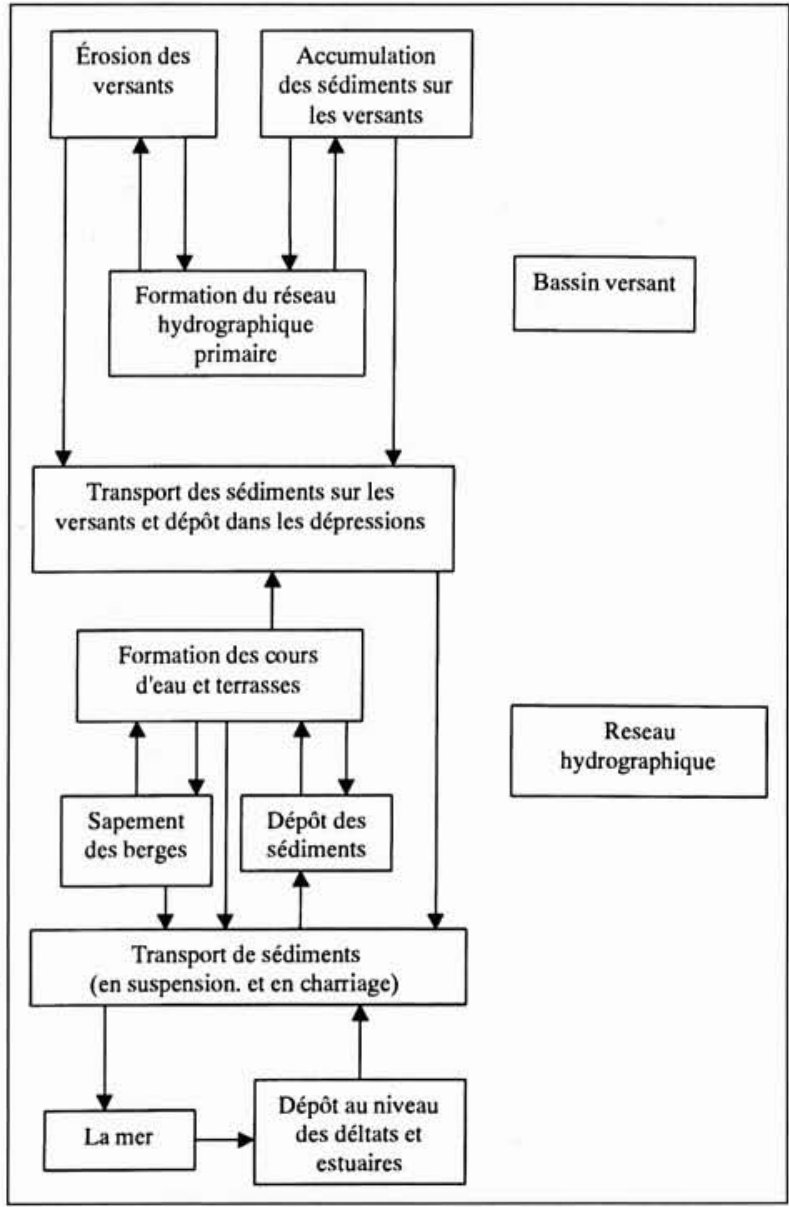

1. Genèse et cheminement des sédiments dans les cours d'eau et bassins versants, d'après Karaouchov [1]. 
Eau : ressources, aménagements, environnement

\begin{tabular}{|c|c|c|c|c|c|c|c|c|}
\hline Barrages & Oued & Ip & Dd & Dh & $\mathrm{Ce}$ & $\mathrm{C}\left(\mathrm{Mm}^{3}\right)$ & $\mathbf{A}\left(\mathbf{M m}^{3}\right)$ & C/A \\
\hline BOUGHZOUL & Chlef & - & - & 1,09 & 0,014 & 55 & 82,8 & 0.66 \\
\hline GHRIB & Chlef & 0,14 & - & 1,49 & 0,020 & 280 & 148 & 1,89 \\
\hline O. FODDA & Fodda & 0,14 & 4,7 & 1,75 & 0,200 & 228 & 82,76 & 2,75 \\
\hline BAKHADDA & Mina & 3,5 & 0,60 & 0,130 & 56 & 70 & 0,80 & \\
\hline S.M.B.A. & Mina & - & - & 1,82 & 0,060 & 235 & 120 & 1,96 \\
\hline HAMIZ & Arbatach & 0,24 & 4,1 & 1,70 & 0,480 & 21 & 52 & 0,40 \\
\hline EGHILMDA & Agrioum & 0,20 & 4,5 & 1,51 & 0,280 & 154 & 175 & 0,88 \\
\hline ZARDEZAS & Safsaf & 0,18 & 3,5 & 1,53 & 0,240 & 31 & 53 & 0,58 \\
\hline CHEFFIA & Bounamo & 0,17 & 3,1 & 1,96 & 0,300 & 171 & 140 & 1,22 \\
\hline KSOB & Ksob & 0,13 & 4 & 1,10 & 0,100 & 31 & 51,5 & 0,60 \\
\hline F. ELGHERZA & Elabiod & - & 5,5 & 1,38 & 0,040 & 47 & 21 & 2,24 \\
\hline F.ELGHEISS & Gheiss & 0,26 & 2,8 & 0,86 & 0,160 & 3 & 11,3 & 0,26 \\
\hline SARNO & Sarno & 0,11 & 1 & 1,00 & 0,070 & 22 & 12 & 2,59 \\
\hline CHEURFA & Makerra & - & 2,6 & 1,65 & 0,018 & 14 & 28 & 0,50 \\
\hline BOUHNIFIA & Elhamam & - & - & 1,28 & 0,040 & 73 & 138,8 & 0,52 \\
\hline FERGOUG & Elhamam & - & - & 1,78 & 0,040 & 18 & 151 & 0,12 \\
\hline DJ. ETTORBA & Guir & - & - & - & 0,060 & 360 & 200 & 1,80 \\
\hline BENI BAHDEL & Tafna & 0,09 & 2,4 & 0,92 & 0,160 & 63 & 73,9 & 0,85 \\
\hline MAFFROUCH & Mafrouch & 0,16 & 2 & 0,34 & 0,270 & 15 & 16 & 0,94 \\
\hline
\end{tabular}

Tableau 1: Caractéristiques hydromorphométriques des systèmes étudiés [I].

sont situés dans des régions sub-humides (dont la pluviométrie est un peu supérieure à $600 \mathrm{~mm}$ ) et un seul en climat désertique avec une pluviométrie moyenne interannuelle de $150 \mathrm{~mm}[1]$.

Le tableau 1 montre l'ensemble des paramètres géométriques et hydromorphométriques.

\section{EVALUATION DES VOLUMES DES SÉDI- MENTS}

Les données de base ont été élaborées par la Compagnie Géophysique de France et traitées par Géokart dans le cadre d'un contrat avec l'Agence Nationale des Barrages. Les mesures bathymétriques ont été réalisées avec embarcation, à l'aide d'un échosondeur qui enregistre en continu la profondeur. Cet échosondeur était synchronisé avec un stadimètre micro-onde. Les travaux portant sur ces relevés se basent sur les points connus d'après la triangulation et la polygonation géodésique.

La comparaison des plans topo-bathymétriques effectués à deux dates différentes donne le volume d'alluvionnement durant la période comprise entre celles-ci.

Les travaux de dépouillement avaient pour but la réalisation de :

- un plan topo-bathymétrique du réservoir effectué à partir des résultats des relevés sur terrain ;
- un graphique de la relation surface en fonction des niveaux d'eau $[S=\mathrm{f}(H)]$;

- la surface du réservoir à la cote $R_{N}$ et à la cote $R_{\max }$ du graphique de la relation capacité en fonction du niveau d'eau $[y=\mathrm{f}(H)]$;

- la capacité du réservoir à la cote $R_{N}$ et à la cote $R_{\max \text {. }}$ en 1986.

La quantification des volumes de sédiments, pris à partir de différentes sources, est donnée dans le tableau 2 ; les résultats obtenus prennent en compte, le cas échéant, le dévasement et la surélévation.

Cet échantillon de 19 barrages pèche par le manque d'uniformité des périodes d'observations, et une distribution spatiale différente d'où l'irrégularité des relevés topobathymétriques. L'envasement moyen obtenu montre des valeurs un peu disparates qui varient dans une fourchette de $0,017 \mathrm{Mm}^{3} /$ an (cas de Mefrouch dont le bassin versant possède la plus faible taille) et $2,490 \mathrm{Mm}^{3} /$ an (Ghrib dont le bassin versant présente la plus importante superficie). Cela a entraîné une perte dans la capacité initiale qui arrive à la limite de la durée de vie pour certains, tel que le barrage Fergoug dont le bassin versant montre une légère végétation. Le barrage Cheffia présente la plus faible perte en capacité ; cela pourrait être dû à l'influence des caractéristiques de son bassin versant côtier couvert d'une végétation assez dense et est accaparé par des forêts de chênes, qui jouent un rôle capital dans la lutte contre l'érosion. 


\begin{tabular}{|c|c|c|c|c|c|c|}
\hline Barrages & $\begin{array}{c}\text { Superficie } \\
\text { du B.V. } \\
\left(\mathbf{k m}^{2)}\right.\end{array}$ & $\begin{array}{c}\text { Capacité } \\
\text { initiale } \\
\left(\mathbf{M m}^{\mathbf{3}}\right)\end{array}$ & $\begin{array}{l}\text { Période de } \\
\text { calcul }\end{array}$ & $\begin{array}{l}\text { Envas. } \\
\text { moyen } \\
\left(\mathrm{Mm}^{3} / \mathrm{an}\right)\end{array}$ & $\begin{array}{c}\text { Capacité } \\
\text { en } 1986 \\
\left(\mathrm{Mm}^{3}\right)\end{array}$ & $\begin{array}{c}\text { Perte en } \\
\text { capacité } \\
(\%)\end{array}$ \\
\hline BOUGHZOUL & 19740 & 55 & $1934 / 1986$ & 0,570 & 20,38 & 62,94 \\
\hline GHRIB & 23300 & 280 & $1939 / 1986$ & 2,490 & 138,57 & 50,51 \\
\hline O. FODDA & 790 & 228 & $1932 / 1986$ & 2,246 & 132,30 & 41,97 \\
\hline BAKHADDA & 1275 & 56 & $1963 / 1986$ & 0,082 & 45,44 & 18,85 \\
\hline S.M.B.A. & 6100 & 235 & $1978 / 1985$ & 1,170 & 227,40 & 3,23 \\
\hline HAMIZ & 137 & 21 & $1935 / 1986$ & 0,155 & 8,49 & 59,57 \\
\hline EGHILMDA & 650 & 154 & $1954 / 1974$ & 1,832 & 120 & 22,07 \\
\hline ZARDEZAS & 346 & 31 & $1977 / 1986$ & 0,450 & 14,78 & 52,32 \\
\hline CHEFFIA & 575 & 171 & $1965 / 1986$ & 0,124 & 168,40 & 1,52 \\
\hline KSOB & 1470 & 31 & $1977 / 1986$ & 0,395 & 12,43 & 59,90 \\
\hline F. ELGHERZA & 1300 & 47 & $1950 / 1986$ & 0,590 & 25,76 & 45,19 \\
\hline F. ELGHEISS & 153 & 3 & $1939 / 1986$ & 0,030 & 1,60 & 46,66 \\
\hline SARNO & 264 & 22 & $1954 / 1986$ & 0,414 & 21,57 & 1,95 \\
\hline CHEURFA & 4015 & 14 & $1882 / 1967$ & 0,061 & 9 & 35,71 \\
\hline BOUHNIFIA & 7850 & 73 & $1944 / 1967$ & 0,913 & 52 & 28,76 \\
\hline FERGOUG & 8274 & 18 & $1970 / 1986$ & 0,881 & 3,90 & 78,33 \\
\hline DJ. ETTORBA & 22000 & 360 & $1967 / 1986$ & 2,290 & 316,40 & 12,11 \\
\hline BENI BAHDEL & 990 & 63 & $1944 / 1986$ & 0,107 & 56,40 & 10,47 \\
\hline MAFFROUCHE & 85 & 15 & $1940 / 1986$ & 0,017 & 14,60 & 2,66 \\
\hline
\end{tabular}

Tableau 2: Quantification de l'alluvionnement des barrages étudiés, [I].

\section{IV — CALAGE DU MODÈLE}

L'information de base recueillie pour le calage du modèle est présentée dans le tableau 1. Le travail consiste à exploiter les résultats des mesures d'expertises effectués et de déterminer les facteurs hydroclimatiques ou physicogéographiques les plus influents sur le taux d'abrasion.

Les facteurs dont dépend l'érosion sont largement connus. Nous avons sélectionné ceux qui sont quantifiables, disponibles ou faciles à déterminer :

- Caractéristiques hydromorphométriques des bassins versants,

- Pluviométrie,

- Apport moyen interannuel,

- Coefficient d'écoulement,

- Rapport capacité du barrage-apport moyen interannuel.

La lithologie et le couvert végétal ont une influence importante sur l'érosion. En fait, ces facteurs que l'on peut étudier ponctuellement (sur parcelles) ne sont pas modélisables à l'échelle d'un bassin versant (hétérogénéité, effet de changement d'échelle). Ils sont globalement intégrés dans les mesures d'envasement et provoquent les dispersions autour des courbes des équations établies plus loin.

Le paramètre exprimant la sédimentation a été normalisé en taux d'abrasion $(T a)$ c'est-à-dire le volume de sédiments mesuré au niveau de la cuvette du barrage pendant une durée donnée et ramené à une unité de surface du bassin pour une année hydrologique moyenne (tableau 3 ).
Le barrage Ighilemda possède un taux d'abrasion maximal dont le bassin versant côtier cumule de fortes précipitations (coefficient d'écoulement considérable), par contre le barrage de Cheurfa présente une valeur importante déterminée par son faible taux d'abrasion. L'impluvium continental de ce dernier est faiblement arrosé (faible coefficient d'écoulement).

La recherche des liens préférentiels entre la sédimentation et les facteurs retenus est effectuée d'abord graphiquement, où le taux d'abrasion (Ta) a été porté sur l'axe des ordonnées et le logarithme de la superficie $(S)$ sur l'axe des abscisses, les valeurs de la deuxième ou de la troisième variable explicative $(C, A, \ldots)$ ont permis la partition de la population étudiée.

Nous avons donc étudié les courbes suivantes :

- Ta en fonction de la surface $(S)$ et la pluviométrie $(P)$,

- Ta en fonction de la surface $(S)$ et l'apport liquide $(A)$,

- Ta en fonction de la surface $(S)$ et successivement tous les paramètres hydromorphométriques,

- Ta en fonction de la surface $(S)$ et le rapport capacitéapport liquide $(C / A)$,

- Ta en fonction de la surface $(S)$ et l'indice d'altitude $(D h)$.

Aucune corrélation n'a été remarquée entre le taux d'abrasion $(T a)$, la superficie $(S)$ et les divers paramètres testés exceptés le coefficient d'écoulement ( $\mathrm{Ce}$, fonction de la lame liquide inter annuelle écoulée) et le coefficient d'altitude $\left(D h=\left(H_{95 \%}-H_{5 \%}\right) / H_{\text {moy }}\right.$, qui ont donné une différen- 


\begin{tabular}{|c|c|c|c|c|}
\hline Barrages & $\begin{array}{c}\text { Superficie } \\
\text { du B.V. } \\
\left(\mathrm{km}^{2}\right)\end{array}$ & $\begin{array}{c}\text { Pluviométrie } \\
\text { interannuelle } \\
(\mathrm{mm})\end{array}$ & $\begin{array}{l}\text { Envas. } \\
\text { moyen } \\
\left(\mathrm{Mm}^{3} / \mathrm{an}\right)\end{array}$ & $\begin{array}{c}\text { Taux } \\
\text { d'abrasion } \\
\left(\mathrm{m}^{3} / \mathrm{km}^{2} / \mathrm{an}\right)\end{array}$ \\
\hline BOUGHZOUL & 19740 & 300 & 0,570 & 28,85 \\
\hline GHRIB & 23300 & 331 & 2,490 & 129 \\
\hline O. FODDA & 790 & 535,5 & 2,246 & 2233 \\
\hline BAKHADDA & 1275 & 430 & 0,082 & 76,23 \\
\hline S.M.B.A. & 6100 & 330,8 & 1,170 & 160,41 \\
\hline HAMIZ & 137 & 790 & 0,155 & 2289 \\
\hline EGHILMDA & 650 & 946 & 1,832 & 2558 \\
\hline ZARDEZAS & 346 & 642 & 0,450 & 1825 \\
\hline CHEFFIA & 575 & 798,5 & 0,124 & 217 \\
\hline KSOB & 1470 & 370,7 & 0,395 & 268 \\
\hline F. ELGHERZA & 1300 & 388,2 & 0,590 & 454 \\
\hline F. ELGHEISS & 153 & 456,6 & 0,030 & 191 \\
\hline SARNO & 264 & 459 & 0,414 & 54 \\
\hline CHEURFA & 4015 & 389 & 0,061 & 16 \\
\hline BOUHNIFIA & 7850 & 460 & 0,913 & 118 \\
\hline FERGOUG & 8274 & 460 & 0,881 & 104 \\
\hline DJ. ETTORBA & 22000 & 150 & 2,290 & 105 \\
\hline BENI BAHDEL & 990 & 450 & 0,107 & 56 \\
\hline MAFFROUCHE & 85 & 688,2 & 0,017 & 205 \\
\hline
\end{tabular}

Tableau 3 : Taux d'abrasion dans les barrages étudiés. tiation nette de dix-neuf barrages répartis en trois groupes bien distincts (figure 2).

La liaison entre la partition typologique obtenue et le contexte géographique des barrages a permis de constater une répartition des barrages en trois classes $\mathrm{Ia}$, Ib et II et ceci en fonction de trois facteurs essentiels : la superficie du bassin versant $(S)$, le coefficient d'écoulement $(C)$ et l'indice d'altitude $(D h)$.

L'analyse en composantes principales, appliquée sur l'ensemble des données des barrages étudiés : 18 individus (barrages) et 5 variables (superficie, taux d'abrasion, rapport entre la capacité du barrage et l'apport moyen interannuel, coefficient d'écoulement, coefficient d'altitude) et présentés dans la figure 2 , a permis de confirmer la répartition manuelle, [3].

La répartition des variables montre que l'axe I oppose le pôle " coefficient d'écoulement, taux d'abrasion ", qui sont

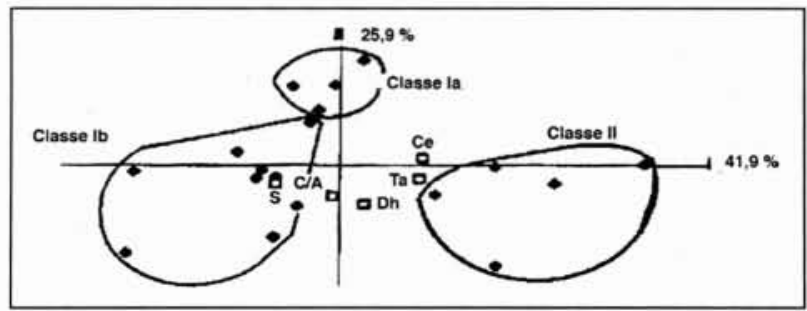

2. Analyse en composantes principales des données des barrages étudiés. Plan factoriel I-II positivement corrélés, au pôle " surface ". Cet axe représente les processus physiques (apports) issus des écoulements en fonction de la superficie, donc il peut être baptisé " effet d'écoulement ". L'axe II est caractérisé par le coefficient d'altitude et un peu par le rapport C/A, et peut représenter le relief des systèmes étudiés.

La distribution des individus (barrages) montre, globalement, trois groupes distincts : le premier groupe, (Oued Fodda, Hamiz, Ighilemda, Zardezas, Cheffia) caractérisé par de fortes valeurs des coefficients d'écoulements et taux d'abrasion, est opposé selon l'axe I ( $41,9 \%$ de variance) au second groupe (Boughzoul, Ghrib, S.M.B.A., Foum Elgherza, Sarno, Cheurfa, Bouhanifia, Fergoug) qui possède les plus grandes tailles de bassin. Et un troisième groupe hétérogène (Bakhadda, Foum Elgheis, Beni Bahdal, Mefrouch) dont les caractéristiques ne présentent aucune tendance générale avec des coefficients d'altitudes moyens.

Les groupes $\mathrm{Ia}, \mathrm{Ib}$ et II obtenus dans le calage graphique apparaissent bien dans les résultats issus de l'A.C.P., et ils sont typiquement bien représentés, [3]. Ces résultats corroborent les constatations formulées précédemment. Et les relations entre les classes de barrages et les facteurs déterminants sont pertinentes.

Après l'analyse manuelle et en composantes principales, on a pu mettre en évidence un regroupement de barrages en trois catégories dépendant essentiellement de la superficie, de l'indice d'altitude et du coefficient d'écoulement, ce qui a permis d'établir le tableau 4. 
Tableau 4 : Classification des barrages étudiés.

\begin{tabular}{c|c|c|c|c|}
\hline Classes & $\begin{array}{c}\text { Superficie } \\
\text { du B.V. } \\
\mathrm{km}^{2}\end{array}$ & $\begin{array}{c}\text { Coefficient } \\
\text { d'écoul. }\end{array}$ & $\begin{array}{c}\text { Indice } \\
\text { d'altitude } \\
\mathrm{m}^{3} / \mathrm{km}^{2} / \mathrm{an}\end{array}$ & $\begin{array}{c}\text { Taux } \\
\text { d'abrasion }\end{array}$ \\
\hline Ia & $<1000$ & 0,1 à 0,3 & $<1$ & $<500$ \\
\hline Ib & $>1000$ & $<0,15$ & 1 à 2 & $<500$ \\
\hline II & $<1000$ & 0,2 à 0,5 & 1,5 à 2 & $>1700$ \\
\hline
\end{tabular}

Classe Ia : Bakhadda, Foum Elgheiss, Beni Bahdel, Maffrouche.

Classe Ib : Boughzoul, Ghrib, S.M.B.A... Ksob, Foum Elgherza, Sarno, Cheurfa, Bouhnifia, Fergoug.

Classe II : Oued Fodda, Hamiz, Cheffia, Eghilmda, Zardezas.

Pour plus de précision, nous avons procédé à des calages provisoires de fonctions basés sur la méthode des moindres carrés [4], les équations testées sont de type linéaire, puissance et exponentiel :

$\mathrm{f}(x)=a x+b ; \mathrm{f}(x)=a x^{n}+b ; \quad \mathrm{f}(x)=a x^{n}+b ; \quad \mathrm{f}(x)=a \mathrm{e}^{r} ;$ $\mathrm{f}(x)=a \mathrm{e}^{-x}+c$

où $x$ est la superficie en $\mathrm{km}^{2}$.

L'ajustement des séries " taux d'abrasion, superficie " sur les résultats d'observation des trois catégories de barrages prédéfinies, en tenant compte de la minimisation de l'erreur par la méthode des moindres carrés, donne les résultats suivants (tableau 5) :

Tableau 5 : Equations du calage par la méthode des moindres carrés.

\begin{tabular}{c|c|c|c|c|}
\hline $\begin{array}{c}\text { Classe de } \\
\text { barrages }\end{array}$ & $\begin{array}{c}\text { Superficie } \\
\mathrm{km}^{2}\end{array}$ & Equation & $\begin{array}{c}\text { Coef. de } \\
\text { corrélation }\end{array}$ & Erreur \\
\hline la & $S<1000$ & $T \mathrm{a}=1433 S^{-0.43}-15,24$ & & $\pm 64,20$ \\
\hline Ib & $S>1000$ & $T a=1846 S-0.15-360$ & 0,853 & $\pm 172,48$ \\
\hline II & $S<1000$ & $T a=0,463 S+2026$ & & $\pm 363,34$ \\
\hline
\end{tabular}

Les meilleurs ajustements donnent des coefficients de corrélation faibles, et des erreurs optimisées assez importantes surtout pour la classe II. Ces résultats indiquent que la superficie seule n'est pas un facteur déterminant, et l'aberration est de ne pas intégrer davantage de paramètres régissants les mécanismes sédimentaires dans la zone d'étude. Donc, le recours aux résultats de l'abaque de classification (graphique et A.C.P.) s'avère plus authentique, [5].

\section{V — CLASSIFICATION ET PRÉDÉTERMINA- TION DE LA DURÉE DE VIE DES BAR- RAGES RÉCENTS}

La première application de cette taxonomie a été faite pour apparenter les barrages récemment mis en service et la prédétermination de leurs durées de vie.

L'identification de ces taxons est une étape fastidieuse mais indispensable pour : l'aménagement des bassins versants par des moyens de lutte contre l'érosion, la meilleure gestion des capacités des barrages et la planification des programmes de dévasement ou de surélévation.

La durée de vie du barrage sera calculée comme le rapport entre $80 \%$ de la capacité et le volume d'envasement moyen, [6]. Les résultats sont donnés par le tableau 6.

La classe de chaque barrage est déterminée dans une première étape par l'étude des caractéristiques physiques et hydrographiques (superficie, coefficient d'écoulement, indice d'altitude). L'ensemble des nouveaux barrages étudiés s'apparentent aux catégories Ib et II. Les valeurs calculées de la durée de vie sont variables. Il convient de signaler que les barrages dont la durée de vie est courte (B. Amrane, S. Yacoub, Deurdeur) sont à aménager en priorité c'est-à-dire mettre au point un protocole d'exploitation bien particulier en vue de synchroniser l'ouverture des vidanges de fond et effectuer des chasses de sédiments, et rechercher d'éventuels courants de densité et surélévation.

Quelques auteurs donnent des modèles de détermination de la durée en supposant la diminution, dans le temps, du

Tableau 6 : Classification et durée de vie des barrages récents.

\begin{tabular}{|c|c|c|c|c|c|c|c|}
\hline Barrages & $\begin{array}{l}\text { Date mise } \\
\text { en service }\end{array}$ & Class & $\begin{array}{l}\text { Volume. } \\
\text { d'env. moy. } \\
\text { (Mm³/an) }\end{array}$ & $\begin{array}{c}\text { Superficie } \\
\left(\mathbf{k m}^{2}\right)\end{array}$ & $\begin{array}{l}\text { Taux } \\
\text { d'abrasion } \\
\left(\mathrm{m}^{3} / \mathrm{km}^{2} / \mathrm{an}\right)\end{array}$ & $\begin{array}{c}\text { Capacité } \\
\text { initiale } \\
\left(\mathbf{M m}^{3}\right)\end{array}$ & $\begin{array}{c}\text { Durée } \\
\text { de vie } \\
\text { (an) }\end{array}$ \\
\hline Deurdeur & 1984 & II & 1,05 & 468 & 2244 & 115 & 88 \\
\hline S. Yakoub & 1985 & II & 2,26 & 920 & 2457 & 264 & 93 \\
\hline Gargar & 1988 & $\mathrm{Ib}$ & 0,57 & 2900 & 197 & 450 & 632 \\
\hline Keddara & 1985 & II & 0,19 & 93 & 2043 & 146 & 615 \\
\hline Bouroumi & 1985 & II & 0,31 & 150 & 2067 & 188 & 485 \\
\hline Guenitra & 1984 & II & 0,43 & 202 & 2129 & 125 & 233 \\
\hline B. Amrane & 1987 & $\mathrm{Ib}$ & 0,66 & 3700 & 178 & 16,5 & 20 \\
\hline Ouzert & 1987 & $\mathrm{Ib}$ & 0,47 & 2100 & 224 & 100 & 170 \\
\hline H. Debagh & 1987 & $\mathrm{Ib}$ & 0,31 & 1070 & 290 & 220 & 568 \\
\hline A. Zada & 1986 & $\mathrm{Ib}$ & 0,47 & 2080 & 226 & 125 & 213 \\
\hline S. Abdelli & 1988 & Ib & 0,32 & 1135 & 282 & 110 & 275 \\
\hline
\end{tabular}




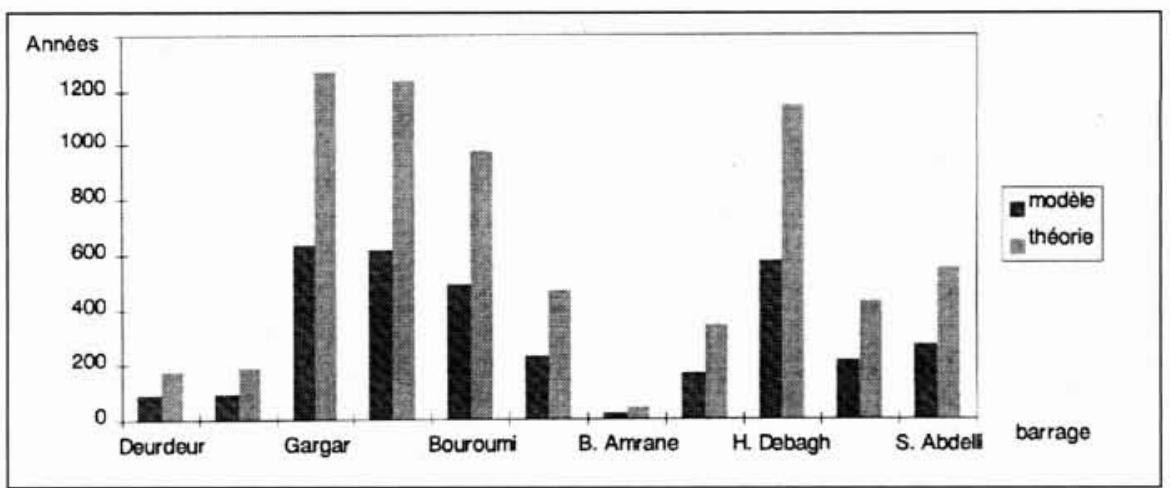

3. Durée de vie des barrages (mis en service entre 1984/1988)

taux d'abrasion à cause du tassement des horizons de vase inférieurs [7]. Or nos corrélations se basent sur un taux d'abrasion moyen, il serait donc intéressant d'étudier l'évolution dans le temps de la vitesse d'envasement pour les barrages possédant plusieurs mesures bathymétriques afin de tester la véracité de ces hypothèses de travail dans des conditions propres à l'Algérie.

La figure 3 montre une comparaison entre la durée de vie théorique et la durée de vie donnée par notre modèle.

On remarque, après une dizaine d'années d'exploitation, que la durée de vie donnée par nos modèles reflète mieux l'état réel actuel de l'envasement des cuvettes de ces ouvrages.

La figure 4 montre l'état actuel de l'avancement de l'envasement du barrage de Beni Amrane (mis en service en 1987) donné par notre modèle et confirmé par la réalité du terrain, [8].

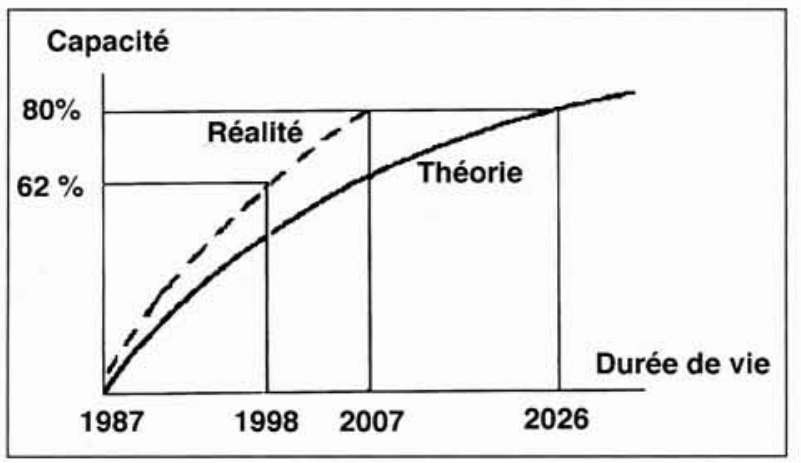

4. Durée de vie du barrage de Beni Amrane (mis en service 1987).

\section{CONCLUSION ET RECOMMANDATIONS}

La mise au point d'une méthodologie simple et pratique pour la quantification de la sédimentation était toujours un objectif attractif. Les résultats des campagnes topo-bathymétriques au niveau de dix-neuf barrages ont permis donc à la fois la partition d'un échantillon et l'utilisation de ses taxons comme classification. Si du point de vue temporel elles ne sont pas homogènes (synchrones), elles ont cependant l'avantage d'intégrer tous les types de sédiments (en suspension, charrié, érosion des berges, dépôts et reprises), et l'inconvénient de ne pas inclure la partie du transport solide déversée pendant les crues [9].

La sédimentation des ouvrages dépend donc essentiellement de la taille du bassin versant, des précipitations, de l'apport liquide (coefficient d'écoulement) et du relief (indice d'altitude). Ce dernier paramètre indique que la majorité des bassins versants sont plus ou moins montagneux, ce qui induit une certaine rudesse du climat avec un effet de continentalité (éloignement de la mer), donc une localisation soit en montagne soit sur les hauts plateaux.

Ce travail est une contribution à l'étude de la sédimentation des barrages situés en zone semi-aride ; il peut être encore enrichi en respectant les propositions suivantes:

- étude systématique des bassins versants de ces ouvrages par modélisation numérique de terrain (M.N.T.),

- élaboration d'un fichier maghrébin d'exploitation des barrages ayant pour objectif d'actualiser les bilans hydrauliques (apports, pertes, évaporation...),

- application d'un protocole d'exploitation rigoureux et standardisé au niveau de ces ouvrages,

- réalisation de campagnes bathymétriques périodiques.

\section{BIBLIOGRAPHIE}

[1] KARAOUCHOV A.V., 1977. Les débits solides, analyse et répartition géographiques. Hydrométeoizdat, Leningrad, 238 p.

[2] KASSOUL M., 1991. Contribution à l'étude de la sédimentation des barrages en Algérie Mémoire fin d'études, Ecole Nationale Supérieure de I'Hydraulique, Blida, 66 p.et annexes.

[3] MUDRY J., 1991. L'analyse discriminante, un puissant moyen de validation des hypothèses hydrogéologiques. Revue sci. de l'eau nº 4 , p.19-37.

[4] BOBEE B., LACHANCE M., POTVIN L., 1981. Analyse multivariée et interprétation géochimique de la variabilité spatiale de la quantité de l'eau et des rivières du Québec méridional. Journal of Hydrology $n^{\circ} 53$, p.95-116.

[5] KASSOUL M., ABDELGADER A., BELORGEY M., 1997 Caractérisation de la sédimentation des barrages en Algérie. Revue sci, de l'eau $n^{\circ} 10(3), p .339-358$.

[6] SOGREAH, ANRH, 1989. Etude méthodologique sur I'hydrologie des retenues colinéaires et mise au point d'outils de calculs à l'usage des directions de l'hydraulique. Rap. tech. $n^{\circ} 5$, Grenoble, $156 \mathrm{p}$.

[7] RAMPON A., 1977 Sédimentation des barrages, érosion et transport sédimentaire, 210 p. France.

[8] KASSOUL M.; BELORGEY M.; 1998 Contribution à l'étude de l'alluvionnement des ouvrages hydrotechniques des pays du Maghreb, 25èmes journées de l'hydraulique, S.H.F., Chambéry. France, Tome 2 pp. 461-472.

[9] JOHNSON G., 1983. Etude de l'érosion et des transports solides en zone semi-aride, envasement et dévasement des retenues en Algérie. Rapport PNUD - SOGREAH-ANRH, 28p. 